\title{
PROBLEM OF UNIQUENESS IN THE RENEWAL PROCESS GENERATED BY THE UNIFORM DISTRIBUTION ${ }^{1}$
}

\author{
D. UGRIN-ŚPARAC \\ ETF, University of Zagreb \\ Department of Mathematics \\ Unska 3, P.P. 148 \\ 41001 Zagreb, CROATIA (Hrvatska)
}

\begin{abstract}
The renewal process generated by the uniform distribution, when interpreted as a transformation of the uniform distribution into a discrete distribution, gives rise to the question of uniqueness of the inverse image. The paper deals with a particular problem from the described domain, that arose in the construction of a complex stochastic test intended to evaluate pseudo-random number generators. The connection of the treated problem with the question of a unique integral representation of Gamma-function is also mentioned.
\end{abstract}

Key words: Renewal process, tests of pseudo-random number generators, piece-wise entire density of exponential type, Gammafunction.

AMS (MOS) subject classifications: Primary $60 \mathrm{~K} 05$; Secondary $33 \mathrm{~A} 15,65 \mathrm{C} 10$.

\section{INTRODUCTION}

New powerful methods for evaluation of pseudo-random number generators were developed in papers [7] and [8]. Here will be sketched the main ideas. If a generator is represented by a sequence of independent random variables $U_{1}, U_{2}, \ldots$, with the continuous uniform distribution $U(0,1)$, whose probability density function is denoted by $\varphi$, then one can consider how many summands $n$ will be needed to achieve $U_{1}+\ldots+U_{n} \leq g$ and $U_{1}+\ldots+U_{n+1}>g$, for a fixed positive real $g$. Of course, $n$ is a value of a random variable, which is denoted here by $Y$. Also, it will be said that $Y$ has $e(g)$-distribution. Thus one arrives at a model of the renewal process generated by the uniform distribution. Repeating the described algorithm, one obtains the output sequence $Y_{1}, Y_{2}, \ldots$, of independent random variables with identical $e(g)$-distribution. However, this renewal process serves here as a transformation of the distribution defined by $\varphi$ into the $e(g)$-distribution. Such a kind of

\footnotetext{
${ }^{1}$ Received: February, 1992. Revised: October, 1992.
} 
transformation, based on the renewal process, with a fixed positive real parameter $g$, is named sequential g-transformation. Extensive investigations of the $e(g)$-distribution are presented in [7], and applications to evaluation of numerous pseudo-random number generators are contained in [8].

Experience shows that small abandoning of the examined input sequence from the assumptions made is strongly emphasized in the output sequence. However, if a distribution different from $U(0,1)$ can produce $e(g)$-distribution by means of the sequential $g$ transformation, then the probability of accepting a wrong hypothesis could be increased. This opens the question of uniqueness of the inverse image of $e(g)$-distribution under the sequential $g$-transformation. A more general and more precise statement of the described problem will be given in the next section. It seems that the renewal process, if interpreted as a sequential $g$ transformation, has a deep property that implies injective mapping of certain class of distributions. Returning to the particular problem treated in the present paper, let us quote the main result: an input sequence of random variables whose density is different from $\varphi$ and is constructed from any finite number of pieces, each one defined by any entire function of the exponential type, if subjected to the sequential $g$-transformation, cannot produce $e(g)$ distribution. The main difficulty in obtaining the complete solution of the problem originates from the nonlinear character of condition (4). In the present paper, this problem is partially solved by use of the ring structure of the set of Borel functions associated with all entire functions of the exponential type, cf. Lemma 5. We also wish to emphasize the connection of our problem with a unique integral representation of the Gamma function, expressed by Proposition 8. It seems that the theory of functions of a complex variable presently suffers from the lack of a theorem which would yield asymptotic behavior of the integral involved in Proposition 8 , in the case when $t \rightarrow \infty$.

\section{RENEWAL PROCESS AS A TRANSFORMATION OF ONE SEQUENCE OF RANDOM VARIABLES INTO ANOTHER}

Consider a sequence $\left(V_{i}\right)_{i}$ of independent and identically distributed continuous random variables, having values in the unit interval $[0,1)$ and the probability density function $f$. The following inductive definition of the new sequence $\left(Y_{i}\right)_{i}$ can be interpreted as an algorithmic presentation of a renewal process generated by the density $f$. Let the real number $g>0$ be fixed,

$$
Z_{m, n}:=V_{m+1}+\ldots+V_{m+n}, X_{0}=0
$$




$$
\begin{gathered}
Y_{i}:=\max \left\{n \mid Z_{X_{i-1}, n} \leq g\right\}, X_{i}:=X_{i-1}+Y_{i}+1 \\
i=1,2, \ldots .
\end{gathered}
$$

The described process can be seen as a transformation of the sequence $\left(V_{i}\right)_{i}$ into the sequence $\left(Y_{i}\right)_{i}$ of independent and identically distributed discrete random variables with the probability function

$$
p_{g}(n)=P(Y=n)=\int_{0}^{g} f^{(n) *}(t) d t-\int_{0}^{g} f^{(n+1) *}(t) d t .
$$

Here $f^{(n) *}$ denotes the $n$-fold convolution, and $Y$ stands for any $Y_{i}$. The proof, that is very simple, may be found for instance in [1, page 36], or [7] and [8]. From the last two papers, we shall quote here only the results relevant to the present discussion.

In the case of the above general algorithm, it will be said that the distribution law of $Y_{i}$ is obtained by the sequential $g$-transformation of the probability density function $f$, representing the distribution law of $V_{i}$.

In order to prepare a theory for treating pseudo-random number generators, we shall assume that the general sequence $\left(V_{i}\right)_{i}$ is now substituted by the particular case $\left(U_{i}\right)_{i}$, where $U_{i}$ has the continuous uniform distribution $U(0,1)$ in the interval $[0,1)$.

As already said, $\varphi$ denotes the probability density function of $U_{i}, i=1,2, \ldots$, and the corresponding Laplace transform is $\Phi(s)=\mathcal{L}[\varphi]=\left(1-e^{-s}\right) / s$. The resulting $Y_{i}, i=1,2, \ldots$, have a probability function that is obtained from (1) by the substitution $f=\varphi$ and the use of Laplace transformation:

$$
p_{g}(n)=\frac{n-g+1}{(n+1) !} \sum_{r=0}^{[g]}(-1)^{r}\left(\begin{array}{c}
n+1 \\
r
\end{array}\right)(g-r)^{n}, g>0 .
$$

If we intend to use at least two consecutive members of $\left(U_{i}\right)_{i}$ to produce one member of $\left(Y_{i}\right)_{i}$, we ought to take $g \geq 1$. It is claimed in [8] that the best tests are obtained with $g=1$, and this condition will be preferred in our paper.

Now bearing in mind the applications in statistics, it is quite obvious that the following question of uniqueness is of great importance.

Let $C D(0,1)$ be the set of all continuous distributions in the unit interval $[0,1)$. In our context it is natural to represent a distribution by the corresponding density function. On the other hand, we have the set $D D(N)$ of all discrete distributions containing random variables that takes on values in the set $N$ of all natural numbers. Then the described sequential $g$ - 
transformation can be interpreted as a particular case of the mapping

$$
S_{g}: C D(0,1) \rightarrow D D(N)
$$

namely $S_{g}(\varphi)=e(g)$.

In reference [7], the following hypothesis has been posed:

$$
S_{g}^{-1}(e(g))=\varphi
$$

Since we have not seen such questions discussed in renewal theory (cf. for instance [2, pp. 63108]), we offer here the following alternative formulation: hypothesis (2) is equivalent to the uniqueness of the inverse image of $e(g)$-distribution under the sequential $g$-transformation.

From [7] one learns that $S_{1}^{-}(e(1))$ does not contain: Simpson distribution, any square parabola-law and any exponential-law distribution.

In the present paper we shall greatly enlarge the class of distribution laws that do not belong to $S_{g}^{-1}(e(g))$, but the complete solution to the problem seems to be very far.

From reference [9] we quote one form of the necessary and sufficient condition for $f \in C D(0,1)$ to satisfy $S_{g}(f)=e(g)$ :

$$
\int_{0}^{g} f^{(m) *}(t) d t=\frac{1}{m !} \sum_{r=0}^{[g]}(-1)^{r}\left(\begin{array}{c}
m \\
r
\end{array}\right)(g-r)^{m}, m=1,2, \ldots .
$$

\section{SOME EQUIVALENT CONDITIONS}

Let $f \in C D(0,1)$ and let the corresponding Laplace transform be $\mathcal{L}[f]=F$. We are reminded that all density functions that differ on a set of Lebesgue measure zero define the same distribution, and the analogous identification guarantees bijection in $\mathcal{L}$-transformation, cf. [3, p. 72]. By theorems from $\mathcal{L}$-transformation we obtain another condition equivalent to (3):

$$
\frac{1}{2 \pi i} \int_{L_{a}} e^{g s} s^{-1} F^{m}(s) d s=\frac{1}{m !} \sum_{r=0}^{[g]}(-1)^{r}\left(\begin{array}{c}
m \\
r
\end{array}\right)(g-r)^{m},
$$

where $m=1,2, \ldots, a>0, L_{a}=\{a+i \omega \mid \omega \in R\}$. Besides the notation of the line $L_{a}$, we use also throughout the paper, $D_{a}=\left\{s \in C \mid \mathscr{B}_{(}(s) \geq a\right\}$. Though the left-hand side in (4) may be interpreted as a nonlinear integral transformation of $F$, we shall greatly facilitate notations if we look at this as a linear transformation of $F^{m}$. So we denote the left-hand side in (4) by $\mathscr{T}_{g}\left[F^{m}\right]$. We are reminded also that $F$ is an entire function on $C$, cf. $[3$, p. 145]. 
Definition 1: Let $F$ be regular in a right half plane. We say that $F$ has the property $P 1$ if there exists $a>0$ and $M>0$ such that $|F(s)|<M /|s|$ for all $s \in D_{a}$, and $D_{a}$ is contained in the domain of regularity of $F$.

Let $f \in C D(0,1)$ and $F=\mathcal{L}[f]$. We quote the theorem from $\mathcal{L}$-transformation: if $f$ is of bounded variation, then $F$ has the property $P 1$.

Lemma 2: Suppose that $f \in C D(0,1), F=\mathcal{L}[f]$ and there exist functions $F_{1}$ and $F_{2}$ such that $F(s)=F_{1}(s)+F_{2}(s) e^{-\beta s}$ where $\beta \geq g, F_{1}$ and $F_{2}$ have the property $P 1$ and consequently there is $D_{a}$ where both functions are regular. Then

$$
\mathscr{T}_{g}\left[F^{m}(s)\right]=\mathcal{T}_{g}\left[F_{1}^{m}(s)\right], m \in N
$$

Proof: By the binomial formula, the left-hand side in (4) becomes the sum of $m+1$ terms, the integrand of the $r$-th term being

$$
s^{-1} F_{1}^{m-r}(s) F_{2}^{r}(s) e^{(g-r \beta) s}, r=0,1, \ldots, m
$$

To those terms with $r>1$ for $\beta \geq g$ and $r \geq 1$ for $\beta>g$, we can apply Jordan's lemma and Cauchy's theorem, to modify the path of integration into the curve which starts at $+\infty$, encircles the point $a$ once in the clockwise direction, and returns to $+\infty$. In this manner, and for $r=1$ and $\beta=g$ directly, it is immediately seen that the line integrals of these terms are zero. This proves the lemma.

Note: We are using Jordan's lemma not in the original form, cf. [10, p. 115], but in a modified form suitable for Laplace transformation, cf. [5, p. 436]. For easier reference we are quoting this statement:

Given a family of circle arcs $C_{R}=\left\{s \in C|| s \mid=R, \mathscr{B}_{(}(s)>a\right\}, R \rightarrow \infty$, on which the function $G(s)$ tends to zero uniformly with respect to arg $s$, as $R \rightarrow \infty$, when $-\frac{\pi}{2}<$ args $<\frac{\pi}{2}$, then for each $t<0$

$$
\lim _{R \rightarrow \infty} \int_{C_{R}} G(s) e^{s t} d s=0 .
$$

Hence, when considering condition (4), at least for distributions satisfying the assumptions posed in Lemma 2 , we may discard all terms of the form $F_{2}(s) e^{-\beta s}$ for $\beta \geq g$. For instance, if $g=1$ and $\Phi(s)=s^{-1}\left(1-e^{-s}\right)$, the term $s^{-1} e^{-s}$ can be discarded. We call this process reduction modulo $e^{-\beta s}, \beta \geq g$. It is important to point out that after each reduction, we are no longer dealing with the $\mathcal{L}$-transform of the density $f$. 
In the sequel, we discuss two possible modifications of condition (4).

Residue method: Assume that the density functions $f \in C D(0,1)$ are of bounded variation, so that $F=\mathscr{L}[f]$ has the property $P 1$, namely $|F(s)|<M /|s|$ for $s \in D_{a}$. Suppose that the complex $z$ lies in the disk $|z|<k / M$, where $0<k<1$. It follows that $|z F(s)|<k /|s|$ with $s$ on the line $L_{a}$. Multiplying (4) by $(-z)^{m}$ and summing over $m \in N$, we get the integral of a uniformly convergent series with respect to $z$ in that disk, hence a condition equivalent to (4) is as follows:

$$
\mathcal{J}_{g}\left[F(s)(1+z F(s))^{-1}\right]=z^{-1}\left\{1-e^{-g z} \sum_{r=0}^{[g]}(g-r)^{r}\left(z e^{z}\right)^{r} / r !\right\},
$$

where $|z|<k / M$. The analytic extension now can enlarge the domain of $z$ where (5) holds. Note an unusual appearance of the function $\Phi$ on the right-hand side of (5) in the case $g=1$. Hence our problem of uniqueness is now modified into the question of unique representation of the function $\Phi$ by the integral in (5) with $g=1$, at least for densities which are of bounded variation. Jordan's lemma may be applied to (5), this time the family of circle arcs reads: $C_{R}=\left\{s \in C|| s \mid=R, \Re_{(}(s)<a\right\}, R \rightarrow \infty$, yielding an expression of $\Phi$ as a finite or infinite sum of residues. Here, with fixed $F$, dependence on $z$ of the right-hand side in (5) is realized through varying positions of poles in the left-hand side, that introduces great difficulties.

Mellin transformation: The discrete functions of $m$ in condition (4) admit interpolation, that is achieved by use of Mellin transformation. We shall describe the results very concisely, since that method reveals the connection of our main problem with the gamma function.

Assume that $f \in C D(0,1), \mathcal{L}[f]=F$ and that $f$ is of bounded variation. Then $F$ has the property $P 1$.

Definition 3: $\quad$ Let $F$ be regular in a right half plane. We say that $F$ has the property $P 2$ if there exists $a>0$ such that $F$ has no zero in $D_{a}$.

A consequence of $P 2$ is that $G=1 / F$ is regular in $D_{a}$, particularly on $L_{a}$. Let $G\left(L_{a}\right)$ be the image of the line $L_{a}$ under the (conformal) mapping $G$.

Definition 4: $\quad$ Let $F$ have the properties $P 1$ and $P 2$ in the half plane $D_{a}, a>0$. We shall say that $F$ has the property $P 3$ if on each finite segment of the negative real axis, the curve $G\left(L_{a}\right)$ has at most a finite number of intersections with this axis.

Starting from expression (5) it is now possible to prove, by means of Mellin transformation, that (4), where $F$ has the property $P 3$, admits interpolation that consists of 
the substitution of $m \in N$ by $t-1 \in C$ and $m$ ! by $\Gamma(t), \Re(t)>0$. The proof comprises a tricky procedure through which possibly infinite number of singularities are excluded form the line $L_{a}$. The proof of this theorem and its use in solving the main treated problem would double the length of the article. However, the statement of the important Proposition 8, quoted at the end of the next section, would not be understandable without the above considerations.

The existing methods of complex analysis seem to be insufficient in treating our problem. Therefore it is reasonable to develop the theory based on conditions in the form (4). That is done in the next section.

\section{ON A CLASS OF FUNCTIONS SATISFYING THE HYPOTHESIS}

Let $E$ denote the set of all entire functions of the exponential type, i.e. of order at most one and if just of order one then of finite type $\sigma$. As usual, any function $\tilde{f} \in E$ equals its Taylor series

$$
\tilde{f}(t)=\sum_{j=0}^{\infty} a_{j} t^{j} / j !
$$

In that context the corresponding $\mathcal{L}$-transform is called the Borel function associated with $\tilde{f}$, and it reads

$$
\tilde{F}(s)=\sum_{j=0}^{\infty} a_{j} / s^{j+1} .
$$

The definition of exponential type, cf. [4, pp. 122-123] implies that there exist constants $\rho>\sigma$ and $K>0$ such that $\left|a_{j}\right|<K \rho^{j}, j=0,1,2, \ldots$. Without proof we shall state the following simple fact.

Lemma 5: The set $L E=\{\tilde{F} \mid \tilde{F}=\mathcal{L}[\tilde{f}], \tilde{f} \in E\}$ of Borel functions associated with all entire functions of the exponential type, supplied with ordinary addition and multiplication, has the structure of commutative ring without unity.

The above lemma represents the fundamental tool to overcome difficulties introduced by the nonlinear character of condition (4).

The order of zero of $\widetilde{F}$ at infinity is called the degree of $\widetilde{F}$ and is denoted by $\operatorname{deg} \tilde{F}=n$. Besides the fact that any entire function restricted to the interval $[0,1]$ is of bounded variation, it is possible to see directly that there is $a>0$, e.g. $a=2 \rho$, such that $|\tilde{F}(s)|<M /|s|$ for $s \in D_{a}$, where $M$ depends on $\rho$ and $n$ only. Hence $\tilde{F}$ has the property Pl. 
Let $\eta$ denote the unity step function: $\eta(t)=0$ for $t<0$ and $\eta(t)=1$ for $t>0$. Let $\tilde{f} \in E$ be non-negative on $[0,1]$. We construct the density $f \in C D(0,1)$ in the usual way: $f(t)=k \tilde{f}(t)(\eta(t)-\eta(t-1))$. The normalizing factor $k$ shall be absorbed by the coefficients $a_{j}$. Note that non-negativeness of the density function implies $a_{n-1}>0$ where $n=\operatorname{deg} \tilde{F}$ (nontrivial proof!). The corresponding $\mathcal{L}$-transform is $F(s)=F_{1}(s)-F_{2}(s) e^{-s}$, where $F_{1}=\widetilde{F}$ and $F_{2}(s)=\mathcal{L}[\tilde{g}(t)]$,

and consequently

$$
\tilde{g}(t)=\tilde{f}(t+1)=\sum_{j=0}^{\infty} b_{j} t^{j} / j !, b_{j}=\sum_{\nu=0}^{\infty} a_{j+\nu} / \nu !
$$

$$
F_{2}(s)=\sum_{j=0}^{\infty} b_{j} / s^{j+1}
$$

where $\left|b_{j}\right|<K e^{\rho} \rho^{j}, j=0,1, \ldots$. Of course, $\tilde{g} \in E$. Thus we are led to the general form of functions that are naturally treated in this paper, namely

$$
F(s)=\sum_{k=0}^{r} F_{k}(s) e^{-\beta_{k} s}
$$

where $r \geq 0$,

$$
F_{k}(s)=\sum_{j=1}^{\infty} b_{k, j} / s^{j},\left|b_{k, j}\right|<K_{k} \rho j,
$$

$j=1,2, \ldots, k=0,1, \ldots, r, 0=\beta_{0}<\beta_{1}<\ldots<\beta_{r}=1$. Though we are not dealing with the original $f_{k}(t)=\mathcal{L}^{-1}\left[F_{k}(s)\right]$, we assume that $f=\mathcal{L}^{-1}[F]$ is normalized to the unit area and is non-negative on $[0,1]$. If $n_{0}=\operatorname{deg} F_{0}$, then analogously to the earlier conclusion, $b_{0, n_{0}}>0$.

This way introduced $f$ is called a piecewise entire density of the exponential type and the set of all such densities is denoted by $\operatorname{PECD}(0,1)$. It can be easily proved that $\mathcal{L}$-images of functions from $\operatorname{PECD}(0,1)$ have the property $P 1$. Then also have the properties $P 2$ and $P 3$, but proofs are omitted, being too long and immaterial for our main theorem. However, we may state without proof the implication: If condition (4) is satisfied for some $F$, $\mathcal{L}^{-1}[F] \in \operatorname{PECD}(0,1)$, then it is satisfied when $m$ is substituted by complex $t, \mathfrak{R}(t)>-1$.

Our main result is contained in the following statement.

Theorem 6: If $f \in P E C D(0,1) \cap S_{1}^{-1}(e(1))$ then $f=\varphi$.

Proof: Let $f$ be with assumed properties and $F=\mathcal{L}[f]$. Then the equivalent condition is expressed by (4) with $g=1$ :

$$
\mathcal{T}_{1}\left[F^{m}(s)\right]=1 / \Gamma(m+1), m \in N
$$


We shall show that the $\mathcal{L}$-transform $F \neq \Phi$ is excluded by asymptotical behavior of (7) when $m \rightarrow \infty$.

Now, by Lemma 2, we immediately exclude the last term of the sum (6), so that all $\beta_{k}<1$, for $k=0,1, \ldots, r-1$. Next we modify that sum into the form

$$
F(s)=F_{0}(s)+e^{-\beta_{1} s} F_{c}(s) .
$$

Let integer $v$ be chosen such that $\beta_{1} v<1$ and $\beta_{1}(v+1) \geq 1$. Then by use of Lemma 2 , we have for all $m \geq v$ :

$$
F^{m}(s)=F_{0}^{m}(s)+\sum_{\mu=1}^{v}\left(\begin{array}{c}
m \\
\mu
\end{array}\right) F_{0}^{m-\mu}(s) e^{-\beta_{1} \mu s} F_{c}^{\mu}(s)\left(\bmod e^{-s}\right) .
$$

Substituting here the expression denoted by $F_{c}$ and completing needed arithmetics, further reduction modulo $e^{-s}$ is possible. After that, two kinds of terms are obtained: The first term is $F_{0}^{m}(s)$ and the remaining terms with

$$
G_{R}=\left(\begin{array}{c}
m \\
\mu
\end{array}\right) F_{0}^{m-\mu}(s) e^{-\beta s} F_{R}(s)
$$

where $0<\beta<1, F_{R} \in L E$ and $F_{0}^{m-\mu} \in L E$. It turns out that out of all terms (9), the most important is that with the lowest $\beta$. It is easily seen that the sought term is

$$
G_{1}=\left(\begin{array}{c}
m \\
1
\end{array}\right) F_{0}^{m-1}(s) e^{-\beta_{1} s} F_{1}(s),
$$

and it is named the principal term. Condition (7) now reads

$$
\mathcal{T}_{1}\left[F_{0}^{m}(s)\right]+\ldots+\mathcal{T}_{1}\left[G_{R}\right]+\ldots+\mathcal{T}_{1}\left[G_{1}\right]=1 / \Gamma(m+1), m \in N .
$$

The logical structure of the proving process just follows the order of terms on the left-hand side of (11). To the notation of the coefficients in the Laurent expansion of $F_{k}$, we sometimes use simplifications.

(i) Let $\operatorname{deg} F_{0}=n$, then we express $F_{0}$ in the form

$$
F_{0}(s)=c s^{-n}(1+\gamma), \gamma=\sum_{j=1}^{\infty} a_{j} / s^{j}
$$

where, as we already mentioned, $c>0$ and $\left|a_{j}\right|<L \rho^{j}, j=1,2, \ldots, L=K_{0} \rho^{n} / c$. Hence

where

$$
F_{0}^{m}(s)=c^{m} s^{-n m}\left\{1+\sum_{k=1}^{m}\left(\begin{array}{c}
m \\
k
\end{array}\right) \gamma^{k}\right\}
$$

$$
\gamma^{k}=\sum_{j=k}^{\infty} a_{k, j} / s^{j}, a_{1, j}=a_{j}, a_{k, k}=a_{1}^{k}
$$


From the theory of gamma function, cf. for instance [10, pp. 245-246, Example 1], or [6, p. 17, formula (5)], one easily derives the following formula

$$
\mathcal{T}_{1}\left[e^{-\beta s} / s^{\nu}\right]=(1-\beta)^{\nu} / \Gamma(\nu+1)
$$

where $\nu \geq 0, \beta \geq 0$. By the use of this formula one obtains

$$
\mathscr{T}_{1}\left[F_{0}^{m}(s)\right]=c^{m}(\Gamma(n m+1))^{-1}\left\{1+\sum_{k=1}^{m}\left(\begin{array}{c}
m \\
k
\end{array}\right) \sum_{j=k}^{\infty} a_{k, j} /(n m+1)_{j}\right\}
$$

where, as usual, $(x)_{p}=x(x+1) \ldots(x+p-1)$. To justify integration of the involved infinite series term by term, one ought to show that for fixed $k$ these series are uniformly convergent with respect to $s \in L_{a}$. This can be proved if one previously specifies the estimate

$$
\left|a_{k, j}\right|<L^{k} \rho^{j}\left(\begin{array}{c}
j-1 \\
k-1
\end{array}\right) \text {. }
$$

Now, by Weierstrass' criterion (also called $M$-test, cf. [10, p. 49]), the uniform convergence is obtained if $a$ is chosen so that $a>\rho$. Therefore we proved the following statement, that is repeatedly used in the sequel.

Lemma 7: Let $F \in L E$ be represented by its Laurent series at infinity and $\beta>0$, $q>0$. Then the product $e^{-\beta s} s^{-q} F(s)$ may be subjected to the transformation $\mathcal{T}_{1}$ term by term, if $a$ in $L_{1}$ is sufficiently large.

To enable limiting process $m \rightarrow \infty$ under the outer sum in (13), we consider the series as infinite even if $m$ is finite, adding the zero terms. Then we apply Weierstrass criterion as above. Here are some details. The general term of the series is brought to the form

$$
B_{k}(m)=\frac{[m]_{k}}{(n m+1)_{k}} \cdot \frac{A_{k}(m)}{k !}
$$

where $[m]_{k}=m(m-1) \ldots(m-k+1)$ and

$$
A_{k}(m)=\sum_{j=k}^{\infty} a_{k, j} /(n m+k+1)_{j-k}
$$

First of all, one shows in the trivial way that $0 \leq[m]_{k} /(n m+1)_{k}<1$ for $m \geq 1, n \geq 1$, and then by estimate (14) that $\left|A_{k}(m)\right|<(L \rho)^{k} e^{\rho}$. Hence we have the estimate

$$
\left|B_{k}(m)\right|<e^{\rho}(L \rho)^{k} / k !, k=1,2, \ldots,
$$

and this proves the needed uniform convergence of the considered series with respect to $m \in[1, \infty)$. We shall use also a particular consequence of the above consideration, namely from 


$$
A_{k}(m)=\sum_{\mu=0}^{\infty} a_{k, k+\mu} /(n m+k+1)_{\mu}=a_{1}^{k}+\sum_{\mu=1}^{\infty} a_{k, k+\mu} /(n m+k+1)_{\mu}
$$

it follows that $A_{k}(m) \rightarrow a_{1}^{k}$ as $m \rightarrow \infty$. Now (13) implies that

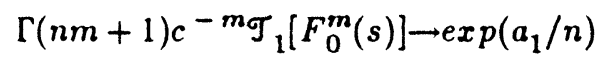

as $m \rightarrow \infty$.

(ii) We now investigate the behavior of the general term $G_{R}$ given by (9), when $m \rightarrow \infty$. Since $F_{R} \in L E$, we have

$$
F_{R}(s)=\sum_{\nu=p}^{\infty} b_{\nu}^{*} / s^{\nu}, p \geq 1,\left|b_{\nu}^{*}\right|<K_{R} \rho_{R}^{\nu}
$$

According to Lemma 5, the following product

$$
(1+\gamma)^{w} F_{R}(s)=\sum_{k=0}^{w}\left(\begin{array}{l}
w \\
k
\end{array}\right) \delta_{k}
$$

where $w=m-\mu$, and

$$
\delta_{k}=\gamma^{k} F_{R}=\sum_{j=k}^{\infty} a_{k, j} / s^{j} \sum_{\nu=p}^{\infty} b_{\nu}^{*} / s^{\nu}=\sum_{\lambda=k+p}^{\infty} b_{k, \lambda} / s^{\lambda},
$$

belongs to the ring $L E$. However, since the exponent $w=m-\mu$ will tend to infinity, we must develop more precise estimates. Let us jump over details and state the final result: assumptions (14) and (17) imply that

$$
\left|b_{k, \lambda}\right| \leq L^{k} K_{R} \rho_{x}^{\lambda}\left(\begin{array}{c}
\lambda-\rho \\
k
\end{array}\right)
$$

where $\rho_{x}=\max \left\{\rho, \rho_{R}\right\}$.

It is clear that dividing the right-hand side of (18) by $\rho_{e}^{\lambda}, \rho_{e}>\rho_{x}$, the new function of $\lambda$ will have the maximum $K_{e}$, which yields the estimate $\left|b_{k, \lambda}\right|<K_{e} \rho_{e}^{\lambda}$. In the same time we demonstrated the main technique used in proving Lemma 5.

Hence we apply Lemma 7 and formula (12) to obtain

$$
\mathscr{T}_{1}\left[G_{R}\right]=\left(\begin{array}{c}
m \\
\mu
\end{array}\right) c^{w}(1-\beta)^{n w}(\Gamma(n w+1))^{-1} \sum_{k=0}^{w} \frac{[w]_{k}}{k !} \frac{A_{k}(n w, p)}{(n w+1)_{p}}
$$

where $w=m-\mu$,

$$
A_{k}(n w, p)=\sum_{\nu=p}^{\infty} \tilde{b}_{k, k+\nu} /(n w+k+1)_{\nu}, \tilde{b}_{k, j}=b_{k, j}(1-\beta)^{j} .
$$

The following estimate is done in two steps. The first one consists of a long calculus and yielding, using (18), that

$$
\begin{gathered}
\left|A_{k}(n w, p)\right| \leq L^{k} K_{R} e^{\tilde{\rho}} \tilde{\rho}^{k+p} /(n w+k+1)_{p} \leq D_{w}(\tilde{\rho} L)^{k} \\
\tilde{\rho}=\rho_{x}(1-\beta), D_{w}=C_{p} /(n w+1)_{p}, C_{p}=K_{R} e^{\tilde{\rho}} \tilde{\rho}^{p}
\end{gathered}
$$


The second step shows how the general term of the outer sum of (19) may be estimated. Its absolute value is less than

$$
D_{w} \frac{[w]_{k}}{(n w+1)_{k}}(\tilde{\rho} L)^{k} / k ! .
$$

Similarly as in section $(i)$, we have $[w]_{k} /(n w+1)_{k} \leq 1$, so that the outer sum is majorized by $D_{w} \exp (\tilde{\rho} L)=C_{p}^{*} /(n w+1)_{p}$, where $C_{p}^{*}=C_{p} \exp (\tilde{\rho} L)$. Now we can prove that

$$
\begin{gathered}
0 \leq \lim _{m \rightarrow \infty} \Gamma(n m+1) c^{-m}\left|\sigma_{1}\left[G_{R}\right]\right| \\
\leq C_{p}^{*} c^{-\mu_{m \rightarrow \infty}} \lim _{m \rightarrow \infty}\left(\begin{array}{c}
m \\
\mu
\end{array}\right)(1-\beta)^{n w}[n m]_{n \mu} /(n w+1)_{p}=0,
\end{gathered}
$$

since $0<1-\beta<1$. This result is true for every term on the left-hand side of (11) except for the first one.

As an obvious and important corollary to the result above, we state that

$$
\lim _{m \rightarrow \infty} P(m) \Gamma(n m+1) c^{-m_{\sigma_{1}}\left[G_{R}\right]=0}
$$

for any polynomial $P(m)$, where $n \geq 1, c>0$.

(iii) Multiply equation (11) by $\Gamma(n m+1) c^{-m}$, call it (11.A) and introduce the notation $X(m)$ for its right-hand side. The logarithm of $X$ is brought into the form

$$
\log X(m)=(n-1) m(\log (m+1)-1)+m(n \log n-\log c)+\sigma(1),
$$

by use of the known asymptotic behavior of gamma function. According to (16) and (20), the logarithm of the left-hand side of the equation (11.A) has the limit value $a_{1} / n$ as $m \rightarrow \infty$. If we assume $n>1$, then $\log X(m) \rightarrow \infty$, which is impossible. Hence $n=1$. Again, if $c \neq 1$, then $\log$ $X(m) \rightarrow \pm \infty$, which also is impossible. Hence $c=1$, and consequently $X(m)=1$ for $m \in N$, which implies that $a_{1}=1$.

(iv) Our initial equation (11) is now slightly simplified:

$$
\sum_{k=1}^{m} \frac{[m]_{k}}{(m+1)_{k}} A_{k}(m) / k !+\sum \Gamma(m+1) \mathcal{T}_{1}\left[G_{R}\right]=0
$$

where $A_{k}(m)$ is given by (15), with $n=1$. Let us emphasize that the second sum in (22) is also finite and comprises the principal term. Multiplying (22) by a polynomial $P(m)$ that will be specified later, we conclude that

$$
\lim _{m \rightarrow \infty} P(m) \sum_{k=1}^{m} \frac{[m]_{k}}{(m+1)_{k}} A_{k}(m) / k !=0,
$$

owing to (21). Let $\operatorname{deg} \gamma=q>1$, so that 


$$
\gamma=\sum_{j=q}^{\infty} a_{j} / s^{j} \text { and } \gamma^{k}=\sum_{j=k q}^{\infty} a_{k, j} / s^{j},
$$

where $a_{k, k q}=a_{q}^{k}$, and one can prove that here

$$
\left|a_{k, j}\right|<L^{k} \rho^{j}\left(\begin{array}{c}
j-1-(q-1) k \\
k-1
\end{array}\right) \text {. }
$$

Therefore this time $A_{k}(m)$ has the form

$$
A_{k}(m)=B_{k}(m) /(m+k+1)_{(q-1) k}, B_{k}(m)=\sum_{j=k q}^{\infty} a_{k, j} /(m+k q+1)_{j-k q} .
$$

As before, we assume the sum in (23) to be infinite. Now we shall majorize this series by a convergent series of constants. In the first place

$$
\left|B_{k}(m)\right|<\left(L \rho^{q}\right)^{k} \sum_{j=0}^{\infty} \rho^{j}\left(\begin{array}{c}
j+k-1 \\
j
\end{array}\right) /(m+k q+1)_{j}<\left(L \rho^{q}\right)^{k} e^{\rho} .
$$

Let us take $P(m)=(m)_{q-1}$, what suffices to derive the obvious fact that

$$
0 \leq \frac{[m]_{k}(m)_{q-1}}{(m+1)_{k}(m+k+1)_{(q-1) k}}<1
$$

for $m \geq 1$ and $k \geq 1, q \geq 2$. The general term of the majorante is $e^{\rho}\left(L \rho^{q}\right)^{k} / k !$, hence the series in (23) is uniformly convergent with respect to $m \in[1, \infty)$. We can accomplish the limiting process term by term. For $k=1$ we have

$$
\lim _{m \rightarrow \infty} \frac{[m]_{1}(m)_{q-1}}{(m+1)_{1}(m+2)_{q-1}} B_{1}(m)=a_{1, q}=a_{q},
$$

however limiting values of all terms with $k>1$ are zero. From (23) we obtain $a_{q}=0$, which is a contradiction. Hence $a_{j}=0$ for $j=1,2, \ldots$, i.e. $F_{0}(s)=1 / s$ and (22) is reduced to

$$
\sum \Gamma(m+1) \mathcal{T}_{1}\left[G_{R}\right]=0 .
$$

(v) In our final step we shall prove that $F_{1}=0$, next $F_{2}=0$, etc. The order of conclusions is dictated by the order of increasing $\beta_{k}$ in (6). The general term of the remainder denoted in (22) by $\Gamma(m+1) \mathcal{T}_{1}\left[G_{R}\right]$ is majorized as in relation (20).

$$
\Gamma(m+1)\left|\mathcal{T}_{1}\left[G_{R}\right]\right| \leq C_{p}^{*}\left(\begin{array}{c}
m \\
\mu
\end{array}\right) \frac{[m]_{\mu}}{(w+1)_{p}}(1-\beta)^{w},
$$

where $w=m-\mu, C_{0}^{*}=K_{R} \tilde{\rho}^{\rho} \exp (\tilde{\rho}(1+L)), \operatorname{deg} F_{R}=p \geq 1$. If the discussed general term differs from the principal term, then $(1-\beta) /\left(1-\beta_{1}\right)<1$ that implies that multiplying (24) by $P(m) /\left(1-\beta_{1}\right)^{m}$, where $P(m)$ is any polynomial in $m$, and taking $m \rightarrow \infty$, there remains something of the principal term only! The principal term, given by (10), now reads

$$
m \Gamma(m+1) \mathcal{T}_{1}\left[s^{1-m_{e}} e^{-\beta_{1} s} F_{1}(s)\right]=m\left(1-\beta_{1}\right)^{m-1} \sum_{j=p_{1}}^{\infty} \tilde{b}_{1, j} /(m+1)_{j-1},
$$

where $\tilde{b}_{1, j}=b_{1, j}\left(1-\beta_{1}\right)^{j}, p_{1}=\operatorname{deg} F_{1}$ and therefore $b_{1, p_{1}} \neq 0$. Now the arbitrary 
polynomial may be specified: $P(m)=(m+1)_{p_{1}-2}$. As we explained, (24) implies

$$
\lim _{m \rightarrow \infty} \sum_{j=p_{1}}^{\infty} \tilde{b}_{1, j}(m)_{p_{1}-1} /(m+1)_{j-1}=0 .
$$

The general term of the sum is majorized by $K_{1}\left(\left(1-\beta_{1}\right) \rho_{1}\right)^{j} / j$ ! with $m \in[1, \infty)$, so the Weierstrass' criterion of uniform convergence enables us to put $m \rightarrow \infty$ under the sum. Thus $b_{1, p_{1}}=0$, a contradiction. Hence $F_{1}=0$. Now the term with $\beta_{2}$ becomes principal, causing $F_{2}=0$. The method works up to the conclusion $F_{r-2}=0$. Now the last term becomes principal, and we need to examine $F_{r-1}$. If the principal term constitutes the whole of the remainder, the method is slightly modified. Then

$$
\begin{gathered}
\left(\begin{array}{c}
m \\
1
\end{array}\right) \Gamma(m+1) \mathcal{G}_{1}\left[s^{1-m} e^{-\beta_{r-1} s} F_{r-1}(s)\right]= \\
m\left(1-\beta_{r-1}\right)^{m-1} \sum_{j=p_{r-1}}^{\infty} \tilde{b}_{r-1, j} /(m+1)_{j-1}=0,
\end{gathered}
$$

for $m \in N, \operatorname{deg} F_{r-1}=p_{r-1}$. Hence the sum is zero for every $m \in N$. The equation may be multiplied by $(m+1)_{p_{r-1}-2} /\left(1-\beta_{r-1}\right)^{m-1}$ and the limit process $m \rightarrow \infty$ accomplished under the summation sign, the series being uniformly convergent with respect to $m \in[1, \infty)$. The result is $b_{r-1, p_{r-1}}=0$, a contradiction. We obtained $F_{r-1}=0$ and therefore $F(s)=F_{0}(s)=1 / s$.

This completes the proof of Theorem 6 .

In the complex analysis approach to our main problem, a unique integral representation of reciprocal gamma function would follow from Theorem 6 . We are quoting that corollary to illustrate the connection of our problem with the theory of gamma functions.

Proposition 8: Let $f \in P E C D(0,1), \mathcal{L}[f]=F$ and let $F$ be reduced modulo $e^{-s}$. Then the statement

implies that $F(s)=1 / s$.

$$
1 / \Gamma(t+1)=\frac{1}{2 \pi i} \int_{L_{a}} e^{s} s^{-1} F^{t}(s) d s, t \in C, \Re_{b}(t)>0, a>0
$$

Remark: As already mentioned, $f \in \operatorname{PECD}(0,1)$ implies that $F$ has the properties $P 1$ and $P 2$ for sufficiently large $a>0$. Then $F^{t}$ denotes the branch which has real values on the part of the real axis contained in $D_{a}$.

The method developed in the proof of Theorem 6 may be easily extended to the case $g>1$. The structure of the general expression on the right-hand side of (4) demands a bit more of skillfulness. The integral in (4) ought to be transformed by the change of integration 
variable: $g s=u$, and then the same steps $(i) \ldots(v)$ should be generalized. The term of the greatest order $g^{m} / \Gamma(m+1)$ cancels and the right-hand side in (22) is no longer zero but a function of $m$ and $g$ which permits the same conclusions as in the case $g=1$. In such a way one can prove the following generalization.

Theorem 9: Let $g$ be fixed and $g \geq 1$. If $f \in P E C D(0,1) \cap S_{g}^{-1}(e(g))$, then $f \in \varphi$.

The usefulness of Theorem 9 becomes more evident, if we are reminded that any continuous function on a finite segment $[a, b]$ permits arbitrary close approximation by Bernstein polynomials, cf. [4, pp. 63-97]. Hence if $f \in C D(0,1)$ has a finite number of continuous segments, then one can find $f^{*} \in \operatorname{PECD}(0,1)$ arbitrary close to $f$ such that $f^{*}$ satisfies hypothesis (2).

\section{REFERENCES}

[1] Cox, D.R., “Renewal Theory”, Methuen, London 1970.

[2] Daley, D.J. and Vere-Jones, D., "An Introduction to the Theory of Point Processes", Springer-Verlag, New York 1988.

[3] Doetsch, G., “Handbuch der Laplace-Transformation”, Birkhäuser, Basel 1950.

[4] Gel'fond, A.O., "Isčislenie Konečnyh Raznostei”, Nauka, Moskva 1967.

[5] Lavrent'ev, M.A. and Šabat, B.A., "Metody Teorii Funkcii Kompleksnogo Peremennogo", 3rd ed., Nauka, Moskva 1965.

[6] Luke, Y.L., "The Special Functions and their Approximation", Vol. I, Academic Press, New York, San Francisco, London 1969.

[7] Ugrin-Sparac, G., "On a distribution encountered in the renewal process based on uniform distribution”, Glasnik Matem. 25 (45), (1990), pp. 221-233.

[8] Ugrin-Šparac, G., "Stochastic investigations of pseudo-random number generators", Computing 46, (1991), pp. 53-65.

[9] Ugrin-Šparac, G., "Some Methods of Investigation of Pseudo-random Number Generators", Doctoral thesis, University of Zagreb 1986.

[10] Whittaker, E.T. and Watson, G.N., "A Course of Modern Analysis", 4th ed., Cambridge University Press 1973. 


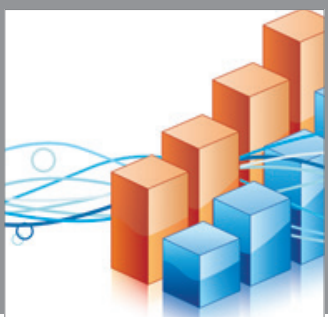

Advances in

Operations Research

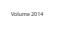

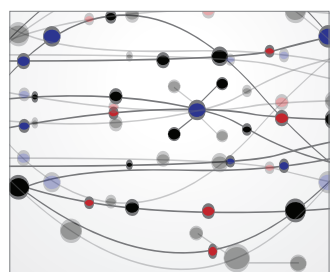

\section{The Scientific} World Journal
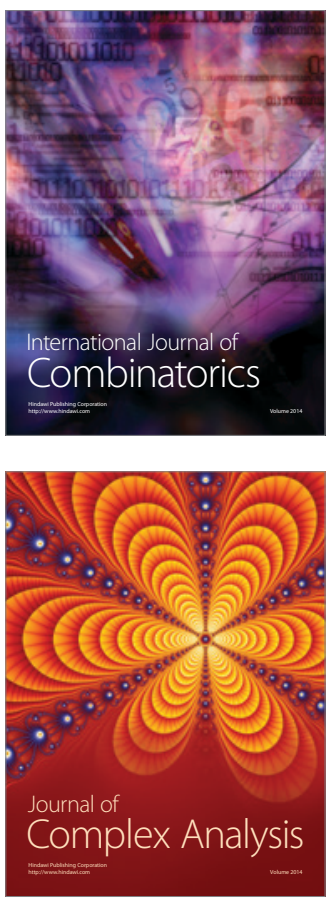

International Journal of

Mathematics and

Mathematical

Sciences
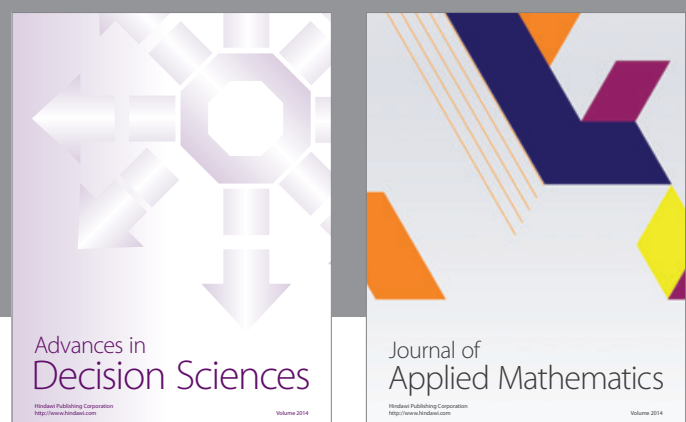

Journal of

Applied Mathematics
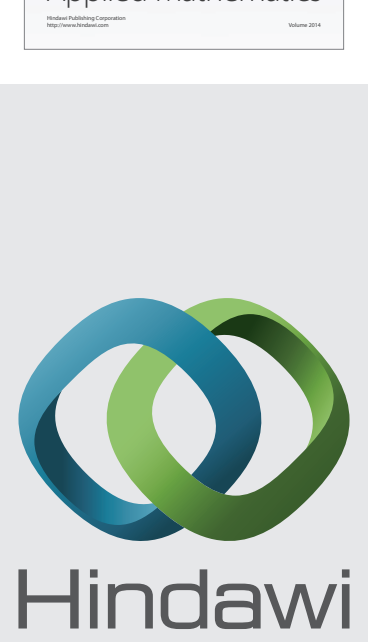

Submit your manuscripts at http://www.hindawi.com
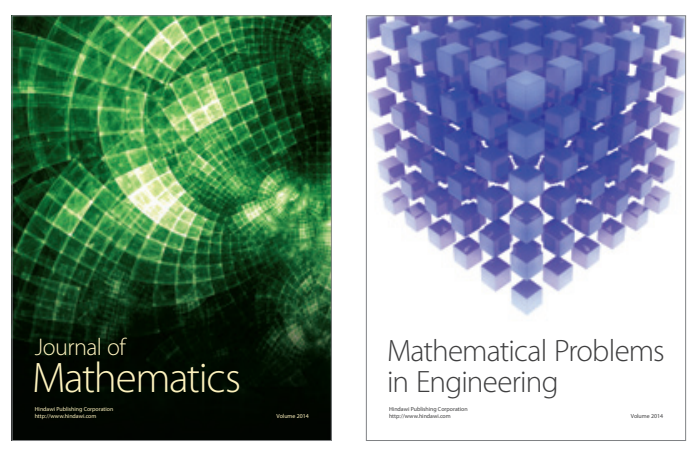

Mathematical Problems in Engineering
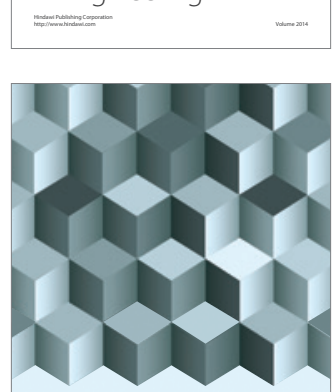

Journal of

Function Spaces
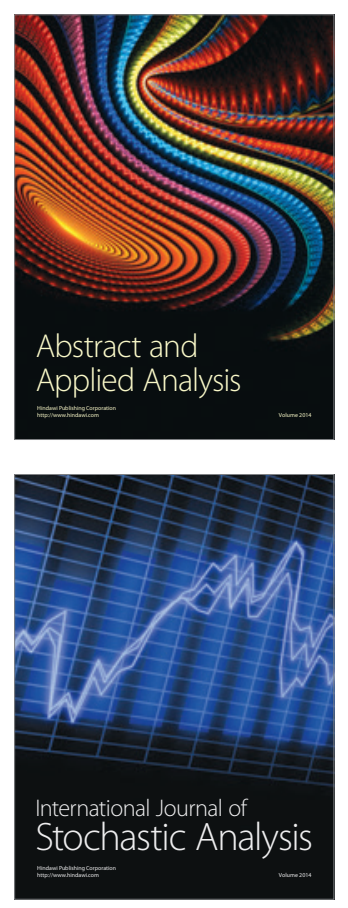

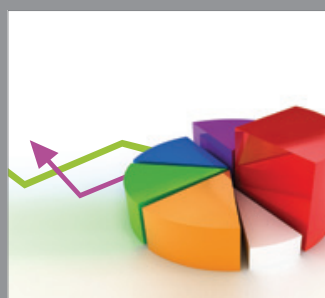

ournal of

Probability and Statistics

Promensencen
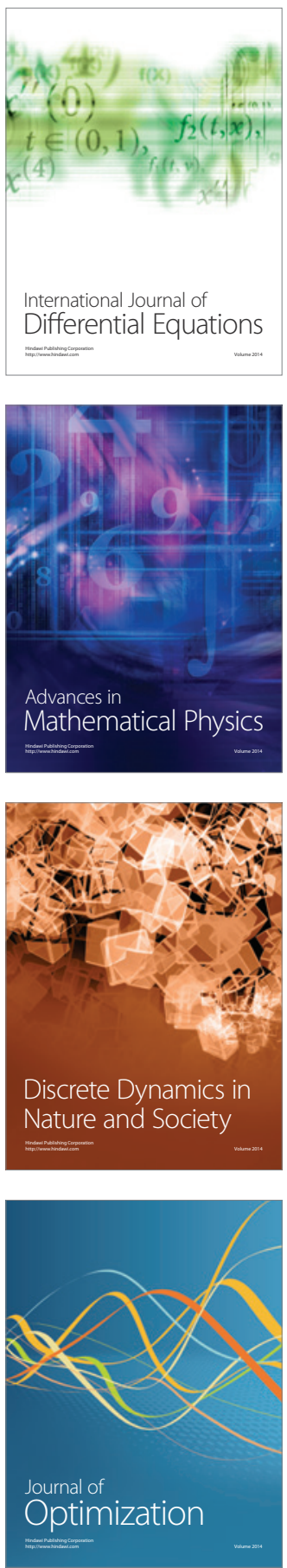